\title{
Modelling the Anatomic Distribution of Neurologic Events in Patients with COVID-19: A Systematic Review of MRI Findings
}

\author{
(D) N. Parsons, (D) A. Outsikas, (D) A. Parish, DR. Clohesy, (D)F. D’Aprano, (DF. Toomey, (DS. Advani, and (D).R. Poudel
}

\begin{abstract}
BACKGROUND: Neurologic events have been reported in patients with coronavirus disease 2019 (COVID-19). However, a modelbased evaluation of the spatial distribution of these events is lacking.
\end{abstract}

PURPOSE: Our aim was to quantitatively evaluate whether a network diffusion model can explain the spread of small neurologic events.

DATA SOURCES: The MEDLINE, EMBASE, Scopus, and LitCovid data bases were searched from January 1, 2020, to July 19, 2020.

STUDY SELECTION: Thirty-five case series and case studies reported 317 small neurologic events in 123 unique patients with COVID-19.

DATA ANALYSIS: Neurologic events were localized to gray or white matter regions of the Illinois Institute of Technology (graymatter and white matter) Human Brain Atlas using radiologic images and descriptions. The total proportion of events was calculated for each region. A network diffusion model was implemented, and any brain regions showing a significant association $(P<.05$, family-wise error-corrected) between predicted and measured events were considered epicenters.

DATA SYNTHESIS: Within gray matter, neurologic events were widely distributed, with the largest number of events ( $10 \%$ ) observed in the bilateral superior temporal, precentral, and lateral occipital cortices, respectively. Network diffusion modeling showed a significant association between predicted and measured gray matter events when the spread of pathology was seeded from the bilateral cerebellum $(r=0.51, P<.001$, corrected) and putamen $(r=0.4, P=.02$, corrected). In white matter, most events $(\sim 26 \%)$ were observed within the bilateral corticospinal tracts.

LIMITATIONS: The risk of bias was not considered because all studies were either case series or case studies.

CONCLUSIONS: Transconnectome diffusion of pathology via the structural network of the brain may contribute to the spread of neurologic events in patients with COVID-19.

ABBREVIATIONS: COVID-19 = coronavirus disease 2019; IIT = Illinois Institute of Technology; NDM = network diffusion model; SARS-CoV-2 = Severe Acute Respiratory Syndrome coronavirus 2

$\mathbf{T}$ he coronavirus disease 2019 (COVID-19) is caused by Severe Acute Respiratory Syndrome coronavirus 2 (SARS-CoV-2). ${ }^{1}$ Typically, patients with COVID-19 present with fever, cough, fatigue, and dyspnea, with approximately $20 \%$ of cases developing severe life-threatening disease. ${ }^{1}$ Extrapulmonary symptoms are also being reported, including altered consciousness, seizures, and focal neurologic injuries, raising concerns of the long-term neurologic sequelae of COVID-19. ${ }^{2-7}$ As of October 15, 2020, >38.4

Received January 14, 2021; accepted after revision February 17.

From the Cognitive Neuroscience Unit (N.P.), School of Psychology (N.P., A.O., A.P., R.C.), and School of Medicine (F.T.), Deakin University, Melbourne, Australia; Melbourne School of Psychological Sciences (F.D.), The University of Melbourne, Melbourne, Australia; Department of Neurology (F.D.), Royal Melbourne Hospital, Melbourne, Australia; Social Behavioural Research Branch (S.A.), National Human Genome Research Institute, National Institutes of Health, Bethesda, Maryland; and Department of Health Sciences (G.R.P.), Mary Mackillop Institute for Health Research, Australian Catholic University, Melbourne, Australia. million cases and 1.1 million deaths have been reported globally, with cases rising rapidly in the United States, India, and Brazil. ${ }^{8}$

Neurologic symptoms in patients with COVID-19 are linked to a broad range of acute neurologic events from large ischemic strokes to small and localized hemorrhages, vascular thrombosis, and microbleeds. ${ }^{9}$ The presence of cerebral microbleeds (small 2- to 5-mm perivascular hemosiderin deposits) are also being reported and are presumed to be features of small-vessel disease. $^{10,11}$ These smaller neurologic events can manifest as

Please address correspondence to Govinda R. Poudel, MD, Mary Mackillop Institute for Health Research, 215 Spring St, Melbourne, Australia; e-mail: govinda.poudel@acu.edu.au; @Govinrp

\footnotetext{
- Indicates open access to non-subscribers at www.ajnr.org

Indicates article with online supplemental data.

http://dx.doi.org/10.3174/ajnr.A7113
} 
FLAIR signal abnormalities in either gray or white matter or localized signal changes as measured with T1-weighted, susceptibility-weighted, and diffusion-weighted MR imaging., ${ }^{9,12,13}$ Most importantly, such neurologic events are known to be associated with long-term impacts on brain function ${ }^{14,15}$ and may therefore reflect a selective vulnerability of brain regions to COVID-19.

These neurologic events are anatomically distributed throughout both cortical and deep subcortical structures. ${ }^{6,16-18}$ However, these distributions are not well-understood and may benefit from mathematic modeling to characterize the pattern of distribution and potential epicenters of spread. For instance, network diffusion models (NDMs) can emulate the pattern of pathologic spread via white matter pathways in the brain and have been useful in modeling the cerebral distribution of pathology in other progressive, degenerative neurologic conditions. ${ }^{19,20}$ However, to date, there has been no application of any mathematic model to assess and map the distribution of neurologic events associated with COVID-19.

This systematic review aims to shed light on the distribution of COVID-19-related neurologic events within gray matter. Subsequently, we summarized recent literature on neurologic events, mapped the spatial distribution of neurologic events, and used the NDM to model the anatomic distribution of gray matter events in patients with COVID-19.

\section{MATERIALS AND METHODS \\ Protocol Registration}

This systematic review was registered with the international prospective register of systematic reviews (PROSPERO: registration No. CRD42020201161) and conducted according to Preferred Reporting Items for Systematic Reviews and Meta-Analyses (PRISMA) guidelines.

\section{Search Strategy}

We searched MEDLINE, EMBASE, Scopus, and LitCovid data bases from January 1, 2020, to July 19, 2020, by "nervous system" OR "CNS” AND "MR imaging" OR "MR imaging" OR "hypointensities" OR "microbleeds" OR “cerebral microbleeds" OR "microhemorrhages." Additional studies were identified by manually searching the reference lists of relevant articles. The search strategy is outlined in the Online Supplemental Data in a PRISMA flow chart. This search was conducted the with help of a health science librarian.

\section{Selection Criteria}

We included case reports, case series, and observational studies published in peer-reviewed journals and preprints available in English that identified small neurologic events in patients with COVID-19 using MR imaging. Articles without full texts and studies in patients without laboratory-confirmed COVID-19 diagnoses were excluded. Any studies that reported only large cerebrovascular events (such as strokes, infarcts) and diffuse pathology (nonspecific) were also excluded.

\section{Data Extraction}

Two independent reviewers screened articles by title and abstract for relevance. These studies were then screened for eligibility for inclusion by full-text evaluation. For each included article, 2 independent reviewers extracted data (A.P., R.C.). Disagreements were collaboratively resolved within the team. Instructions detailing the type of information to be extracted and how to record, categorize, or code this information were also discussed among team members. The following information was extracted from each article: 1) country, first author, and year of publication; 2) sample characteristics (sample size, age group, and sex distribution); 3) study design; 4) clinical symptoms; 5) reason for brain imaging; 6) type of MR imaging performed; 7) imaging findings; and 8) relevant conclusions to assist article interpretation. Two additional reviewers (F.D., F.T.) then validated all the extracted data and the eligibility of each included article.

\section{Neuroimaging Data Synthesis and Coding}

Two expert reviewers (N.P., G.R.P.) screened each included article to identify the location, distribution, and number of neurologic events. These events ranged from microbleeds (observed in SWI or $\mathrm{T}^{*}$ gradient recalled-echo images), white matter hyperintensities (FLAIR images), small lesions, or signal changes in diffusion-weighted imaging within the gray or white matter. For each article, events were manually localized to gray or white matter regions on the basis of available MR images and/or radiologic descriptions. The Desikan-Killiany gray matter atlas incorporating 84 brain regions was used to label any events located within the gray matter. Of the 41 gray matter regions that were impacted, $\sim 70 \%$ of the regions had an exact match with the labels used in the atlas (eg, putamen, caudate, cerebellum, precentral/ postcentral gyri, and so forth). The remaining labels that did not directly match or were broad (eg, basal ganglia, occipitoparietal, temporal, frontal) were localized using the combination of description and MR images available (based on agreement between 2 neuroimaging experts and 1 physician; Online Supplemental Data). White matter bundles from the Illinois Institute of Technology (IIT) Human Brain Atlas were used to label any events located within the white matter. The FSLeyes neuroimaging software from the FMRIB Software Library (https://fsl.fmrib.ox.ac.uk/fsl/fslwiki/FSLeyes) was then used to visualize white matter tracts and gray matter areas from the IIT atlas. Any patient with COVID-19 with nonspecific neuropathologic findings (eg, juxtacortical white matter) or without an accompanying MR image or description was excluded from further analyses. This data-encoding process generated 2 tables for gray and white matter regions with columns corresponding to each article and rows corresponding to the name of the region/ bundle (Online Supplemental Data). Each cell in the table provided information on the number of cases corresponding to a localized neurologic event. A third independent reviewer (F.T.) validated the encoded data, and any discrepancies were discussed and addressed.

\section{Neuroimaging Data Visualization}

The proportion of events $\left(\frac{\text { number of events } \times 100}{\text { total cases }}\right)$ pertaining to each encoded region within the IIT Desikan-Killiany gray matter atlas and IIT white matter bundles was used for visualization. Gray matter events were visualized using MRIcroGL software (https://www.nitrc.org/projects/mricrogl/). White matter events 

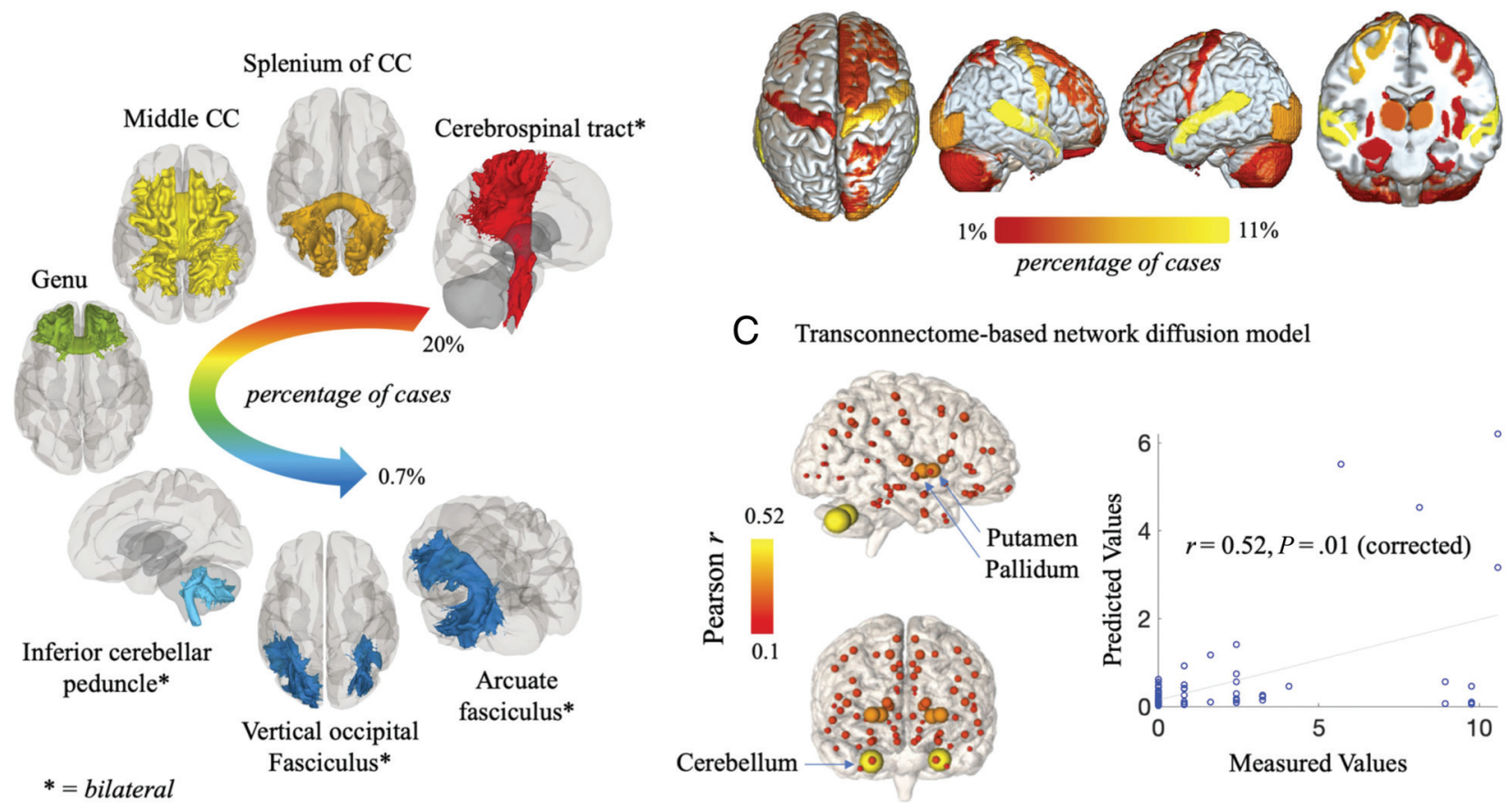

C Transconnectome-based network diffusion model
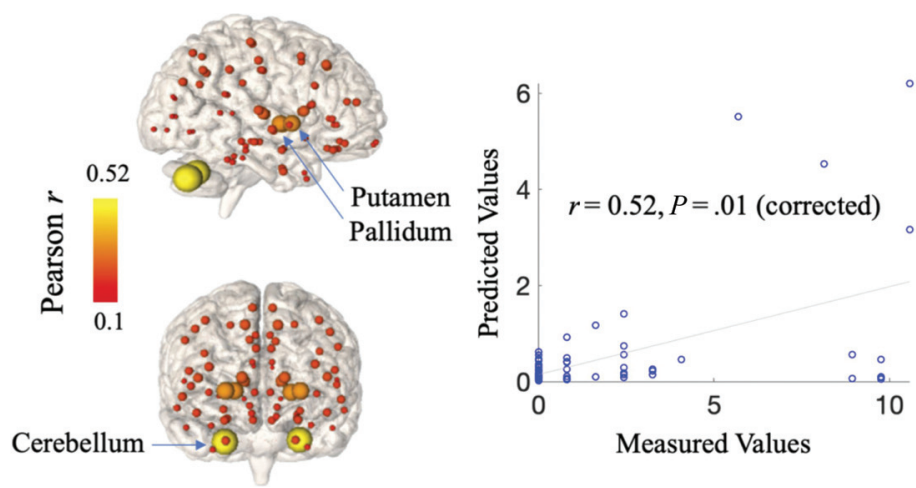

FIG 1. Visualization of the spatial distribution of neurologic events and network diffusion modeling. $(A)$ The highest proportions of events occur in white matter areas such as the corticospinal tract. $(B)$ In gray matter, the most affected regions are the bilateral superior temporal cortices, precentral cortices, and pallidum. (C) A network diffusion model using structural connectivity edge weights successfully predicts the spread of neurologic events. (C) The epicenters of spread that showed the most significant association between predicted and measured distribution of events are the bilateral cerebellum and putamen. CC indicates corpus callosum.

were visualized using Matlab 2018a (MathWorks) and the CONN toolbox, Version 19b (https://web.conn-toolbox.org/).

\section{Network Diffusion Model of Spread}

A graph theoretic meta-analysis model was used to test whether the spatial distribution of small neurologic events in the brain can be explained by a spread via the structural connectome of the brain (source code available at: https://github.com/govin2000/ covidspread). NDM was used per previous protocols that identified a spatial pattern of pathology in the brain. ${ }^{19,20}$ The NDM models the hypothetic distribution of pathology in a brain network (given by a connectome $\mathrm{C}$ ) across time by linear diffusion, given by $x(t)=e^{-\beta H t} x_{0}$, where $x_{0}$ is the initial pattern of the neurologic events at $t=0, H$ is the degree normalized graph Laplacian, and is a diffusivity constant. The unit of the model's diffusion time $(t)$ is assumed to be days (given the likely progression of 5-14 days) for the diffusivity constant of 1 per day. $x(t)$ is a vector of distribution of pathology in the brain when diffusion is seeded from a given region provided by an initial condition $x_{0}$. We used a repeat seeding approach, which has previously been used to identify potential epicenters of the spread of neuropathology. The IIT $(84 \times 84)$ connectivity matrix was used for the NDM simulation.

The NDM generates a vector of distribution of pathology $x(t)$ across time. We expect that $x(t)$ should correlate with the distribution of neurologic events. Thus, the Pearson correlation coefficient strength and $P$ values were calculated between the empiric proportions of events measured using the systematic review method (described above) and $x(t)$ at all model timepoints $(t)$. This process was repeated for all bilateral regions (42 bilateral regions) within the IIT Desikan-Killiany gray matter atlas. The region that showed the largest significant $(P<.05$, family-wise error-corrected for 84 regions) association with measured neurologic events was defined as the seed region.

\section{RESULTS}

The systematic search yielded 461 articles, of which 62 were eligible for full-text assessment (Online Supplemental Data; PRISMA flow diagram). Of these, 28 were excluded; these were commentaries, response letters, and review articles proposing SARS-CoV-2 nervous system invasion but lacking clinical findings. A total of 35 publications reporting small neurologic events in patients with COVID-19 were evaluated. Of these, 35 provided specific anatomic detail required for meta-analysis and modeling. These articles contributed 123 unique patients, with a total of 317 neurologic events (Online Supplemental Data). Of these, 91 patients had gray matter changes, 95 patients had white matter changes, and 72 patients had confirmed cerebral microbleeds. Further details on the presentation of gray and white matter events, and the frequency of symptoms can be found in the Online Supplemental Data.

\section{Spatial Distribution of Neurologic Events}

Figure $1 A,-B$ depicts the spatial distribution of white and gray matter neurologic events. White matter events were observed within 11 of 42 white matter bundles from the IIT atlas. The highest percentage (26\%) of events was observed within the bilateral 
corticospinal tracts, composed of white matter fibers that connect the primary motor cortex and basal ganglia. The splenium and middle of the corpus callosum were affected in $14 \%$ and $9 \%$ of the cases, respectively. The remaining tracts showed white matter events in $<9 \%$ of cases. Of the cerebral microbleeds observed, a similar pattern emerged, in which the largest proportion of cerebral microbleeds was also found in the middle corpus callosum, followed by the splenium of the corpus callosum. Gray matter events were spatially distributed among 41 brain regions within the Desikan-Killiany atlas. The highest proportions $(\sim 10 \%)$ of events were observed in the bilateral superior temporal, precentral, and lateral occipital cortices. Subcortical events were most frequently identified in the pallidum.

\section{Network Diffusion Model Findings}

Each of the 84 regions within the IIT Desikan-Killiany gray matter atlas was used as a potential seed for the spread of pathology across time. Figure $1 C$ shows a glass-brain visualization of the best fit (maximum Pearson correlation coefficient value) between empiric events and predicted values determined using the NDM. A significant fit was achieved when seeding the spread from the bilateral cerebellum (Pearson $r=0.52, P<.001$, corrected) and the putamen $(r=0.4, P=.02$, corrected). Other basal ganglia structures also showed moderate associations $(r>0.3)$ but were not significant after correction. The spatial distribution of the fit in all regions reflects the consequence of network spread, originating from each of the included regions. The association between empiric events and predicted events was low $(r<0.2)$ when the Euclidian distance between regions was used as network edges instead of structural connectivity (Online Supplemental Data).

\section{DISCUSSION}

Patients with COVID-19 are vulnerable to acute neuropathology, commonly in the form of small neurologic and cerebrovascular events. We systematically reviewed articles reporting localized MR imaging findings in patients with COVID-19 and spatially encoded them onto a common gray and white matter atlas. We then investigated whether the spatial distribution of these events follows a cortical or subcortical pattern that can be explained by a linear diffusion-based model of pathologic spread. We found the epicenters of this spread to be the cerebellum and putamen.

\section{Neurologic Events in White and Gray Matter}

White matter events were identified most frequently in the corticospinal tract and corpus callosum. The corticospinal tract is a major white matter pathway connecting critical subcortical brain regions such as the basal ganglia and thalamus and, thus, facilitates information related to voluntary motor control. As a result, diffuse aberrations in the corticospinal tract are associated with motor symptoms such as tendon reflexes, ankle clonus, and bilateral extensor plantar reflexes, which have been commonly reported in patients with COVID-19. ${ }^{21}$ Similarly, the corpus callosum plays an important role in interhemispheric communication, which can result in a disconnection syndrome and broad neurocognitive deficits. ${ }^{22}$
In gray matter regions, events were identified most frequently in the temporal and precentral gyrus as well as the bilateral thalamus. Alterations in thalamocortical connectivity can disrupt the regulation of consciousness and arousal. ${ }^{23}$ Thus, acute events in these regions may explain symptoms such as confusion, disorientation, agitation, and loss of consciousness. ${ }^{10,24}$ Despite their acute manifestation, the accumulation of neurologic events in subcortical structures and consequent disruption to distal cortical regions can increase susceptibility to cognitive impairment and decline, which have ramifications for long-term cognitive prognosis. $^{25,26}$

\section{Infiltration and Spread Mechanism: Olfactory Pathway}

Patients with neurologic symptoms also presented with anosmia, encephalopathy, seizures, and changes in vision including cortical blindness and visual confabulation. ${ }^{27-29}$ Alterations in olfaction may therefore have a neurologic basis, particularly in light of the identified pathology implicating the olfactory bulb in clinical imaging, including the presence of microbleeds among these patients. It is plausible that these symptoms relate closely to the mode of infiltration of SARS-CoV-2, with a potential mechanism being direct injury to the nervous system via angiotensin-converting enzyme 2 receptor expression on nerve cells, including the olfactory bulb.

Given the potential for SARS-CoV-2 neurotropism, neuropathology in the piriform cortex could be caused initially by the introduction of a virus through a direct axonal connection with the olfactory bulb. Although we were not able to mathematically model the piriform cortex specifically within the DesikanKilliany brain atlas, we found only 7 patients from 3 studies who presented with small lesions within this cortex (olfactory bulb). Indeed, some have found intranasal infection of other coronaviruses such as SARS-CoV in the respiratory tract, which were subsequently neuroinvasive. ${ }^{30}$ However, this mechanism remains to be established in the newly discovered SARS-CoV-2.

\section{Infiltration and Spread Mechanism: Axonal Transport}

An important question arises from our finding of the cerebellum and putamen as the seeds of spread-that is, how likely is it that these are indeed epicenters? Herein, note that NDMs are high-level generative models and only model the macroscopic consequences of the pathology in gray matter regions, and that the NDM does not require that the seed region itself presents with the highest number of pathologic events. Indeed, a neurobiologic explanation for the identification of these seed regions is beyond the scope of this article. Therefore, the mechanism underpinning the potential spread from these sites and subsequent distribution of SARS-CoV-2 throughout the brain remains to be thoroughly examined. However, while the cerebellum and putamen may serve as epicenters, SARS-CoV-2 may then travel to cortical sites such as the precentral gyrus via retrograde transsynaptic transmission through the corticospinal tract by hijacking axonal transport mechanisms. ${ }^{22}$ While in transit, direct neuronal or endothelial cell disruption may exacerbate the systemic pathophysiology, facilitating cerebrovascular complications and mixed type I/II respiratory failure. ${ }^{3,22}$

Indeed, the first site of infection of other SARS-CoV viruses is the epithelial cells of the respiratory tract, where they bind to 
angiotensin-converting enzyme 2 receptors; ${ }^{31}$ however, angiotensin-converting enzyme 2 is also expressed on nerve cells. Therefore, coronaviruses may easily be transported to infect the endothelial cells of the blood-brain barrier. ${ }^{31}$ However, SARSCoV-2 has rarely been isolated from CSF samples, precluding the characterization of its neurotropism and direct role in neurologic event pathogenesis.

\section{Neuroinflammation and Cytokine Storm}

Other proposed pathologic mechanisms may explain the distribution of neurologic events, including neuroinflammatory responses and cytokine- and hypoxia-induced injury. ${ }^{3}$ Emerging evidence is characterizing COVID-19 as a vascular disease, a hyperinflammatory response with an ensuing cytokine storm and coagulopathy that may synergistically contribute to neurologic event pathogenesis. ${ }^{2,3}$ COVID-19-associated coagulopathy occurs proportional to disease severity and leads to treatment-resistant thrombotic and hemorrhagic events, characterized by $\mathrm{D}$-dimer elevation with prothrombin prolongation and thrombocytopenia.

Furthermore, cytokine- and hypoxia-induced injury to the corpus callosum, particularly the splenium, has been reported in critical illness, including acute respiratory distress syndrome and highaltitude cerebral edema, potentially contributing to a vulnerability in COVID-19. ${ }^{17}$ Hypoxia directly induces chemical and hydrostatic endothelial cell disruption, promoting vascular permeability and hence contributing to neurologic event pathogenesis. ${ }^{32}$ Relative to the cortex, the thalamus, basal ganglia, and deep white matter are poorly perfused due to their watershed end-arterial vascular architecture, which could exacerbate their baseline hypoxic vulnerability and ultimately promote subcortical neurologic events. While the pathogenesis of white matter hyperintensities remains under debate, roles for hypoxia, immune activation, endothelial cell dysfunction, and altered metabolism have been posited, not dissimilar to the neuropathologic associations of COVID-19. ${ }^{15,18,33}$

\section{Clinical Relevance}

In addition to their acute manifestations, the accumulation of neurologic events in subcortical structures and consequent disruption of distal cortical regions may lead to microstructural injury, independently contributing to cognitive impairment and dementia - a clinically impacting patient morbidity. ${ }^{11,34}$ Microbleeds and white matter hyperintensities also signify a hemorrhage-prone brain, which is more susceptible to larger neurologic events such as ischemic stroke and intracranial hemorrhage, whereby patients demonstrate worse deterioration from premorbid functioning and increased mortality. ${ }^{17}$ Indeed, in patients with COVID-19, the presence of small neurologic events has been associated with increased disease severity, the length of hospital admission, mortality, and worse functional status on discharge. ${ }^{35}$ Given the established adverse effects of small neurologic events and these early findings, the potential long-term cognitive and cerebrovascular impact on patients with COVID-19 may become more apparent with time. If found, careful consideration should be given to the prescription of anticoagulation, given that these neurologic events suggest a propensity for further hemorrhage.

\section{Limitations}

This review has several important limitations. First, we translated neurologic events into a standard MR imaging atlas space using a partly qualitative method, whereby these pathologies were localized using the radiologic description of the location or MR images when available. While this method may lack specificity, we used multiple neuroimaging and medical experts and included only data with specific spatial information or MR images. Thus, the partly qualitative nature of the translation should be considered with caution while interpreting our findings. Furthermore, the white matter tracts used to visualize the summary of the white matter findings are only coarse grain reorientations. Given that few tracts (eg, corpus callosum) completely match the labels used in the atlas, we localized events on the basis of the descriptions and available MR images regarding approximate corresponding tracts. Second, most of the included articles were cross-sectional case studies and hence cannot directly attribute the observed neuropathology to SARS-CoV-2.

The question remains as to whether neurologic events are the direct consequence of viral infection/sequalae or instead indicate an underlying propensity for neurologic events in these patients. ${ }^{36}$ To answer this question, studies must use large samples in which a specific etiology can be isolated in a case-control, longitudinal design. ${ }^{36}$ These etiologies must be separated into those of unrelated vascular origin and those that may be a direct consequence of viral infection. Therein, an additional challenge ensues: to determine whether there are, indeed, overlapping or isolated pathologies in patients with COVID-19. This question could be approached by including patients with previous MR images, in whom existing pathology can essentially be modelled out. We acknowledge that some of the neurologic events included in our study may be explained by the healthy ageing process, whereby white matter hyperintensities are correlated with age. ${ }^{14}$ Furthermore, IIT tractography data were used for visualization of abnormalities in the white matter. Because only a few labels (eg, corpus callosum) completely match the names of the tracts, the locations are only approximate. Hence, the findings regarding white matter changes, for example, white matter hyperintensities in the centrum semiovale, should be interpreted with caution.

\section{CONCLUSIONS}

Patients with COVID-19 exhibit acute neuropathologic and cerebrovascular events. These events occur predominantly in white matter tracts such as the corticospinal tract and corpus callosum, as well in gray matter areas such as the pallidum, putamen, thalamus, and cerebellum. These aberrations likely contribute to altered thalamocortical connectivity and may disrupt the regulation of consciousness and arousal. The accumulation of these events in subcortical structures and the consequent disruption to distal regions may ultimately increase susceptibility to cognitive impairment and decline-having significant long-term cognitive ramifications. Given the prevalence and severity of these manifestations, clinicians should consider having a low threshold for investigating neurologic symptoms and monitoring potential long-term sequelae in patients with COVID-19. 


\section{REFERENCES}

1. Grant MC, Geoghegan L, Arbyn M, et al. The prevalence of symptoms in 24,410 adults infected by the novel coronavirus (SARS-CoV-2; COVID-19): a systematic review and meta-analysis of 148 studies from 9 countries. PLoS One 2020;15:e234765 CrossRef Medline

2. Aghagoli G, Gallo Marin B, Katchur NJ, et al. Neurological involvement in COVID-19 and potential mechanisms: a review. Neurocrit Care 2020 Jul 13. [Edpub ahead of print] CrossRef Medline

3. Ellul M, Benjamin L, Singh B, et al. Neurological associations of COVID-19. Lancet Neurol 2020;19:767-83 CrossRef Medline

4. Mao L, Jin H, Wang M, et al. Neurologic manifestations of hospitalized patients with coronavirus disease 2019 in Wuhan, China. JAMA Neurol 2020;77:683-90 CrossRef Medline

5. Varatharaj A, Thomas N, Ellul MA, et al. CoroNerve Study Group. Neurological and neuropsychiatric complications of COVID-19 in 153 patients: a UK-wide surveillance study. Lancet Psychiatry 2020;7:875-82 CrossRef Medline

6. Radmanesh A, Derman A, Lui YW, et al. COVID-19-associated diffuse leukoencephalopathy and microhemorrhages. Radiology 2020;297: E223-27 CrossRef Medline

7. Kremer S, Lersy F, de Seze J, et al. SFNR-COVID Group. Brain MRI findings in severe COVID-19: a retrospective observational study. Radiology 2020;297:E242-51 CrossRef Medline

8. WHO Coronavirus (COVID 19) Dashboard. Covid19.who.int. https://covid19.who.int/. Published 2020. Accessed October 15, 2020

9. Katal S, Balakrishnan S, Gholamrezanezhad A. Neuroimaging findings in COVID-19 and other coronavirus infections: a systematic review in 116 patients. J Neuroradiol 2021;48:43-50 CrossRef Medline

10. Kremer S, Lersy F, Anheim M, et al. Neurologic and neuroimaging findings in COVID-19 patients: a retrospective multicenter study. Neurology 2020;95:e1868-82 CrossRef Medline

11. Hernandez-Fernandez F, Valencia HS, Barbella-Aponte RA, et al. Cerebrovascular disease in patients with COVID-19: neuroimaging, histological and clinical description. Brain 2020;143:3089-3103 CrossRef Medline

12. Politi LS, Salsano E, Grimaldi M. Magnetic resonance imaging alteration of the brain in a patient with coronavirus disease 2019 (COVID-19) and anosmia. JAMA Neurol 2020;77:1028-29 CrossRef Medline

13. Kandemirli SG, Dogan L, Sarikaya ZT, et al. Brain MRI findings in patients in the intensive care unit with COVID-19 infection. Radiology 2020;297:E232-35 CrossRef Medline

14. Debette S, Markus $H$. The clinical importance of white matter hyperintensities on brain magnetic resonance imaging: systematic review and meta-analysis. BMJ 2010;341:c3666 CrossRef Medline

15. Wardlaw JM, Valdés Hernández MC, Muñoz -Maniega S. What are white matter hyperintensities made of? Relevance to vascular cognitive impairment. J Am Heart Assoc 2015;4:e001140 CrossRef Medline

16. Fitsiori A, Pugin D, Thieffry C, et al. Unusual microbleeds in brain MRI of Covid-19 patients. J Neuroimaging 2020;30:593-97 CrossRef

17. Cannac O, Martinez-Almoyna L, Hraiech S. Critical illness-associated cerebral microbleeds in COVID-19 acute respiratory distress syndrome. Neurology 2020;95:498-99 CrossRef Medline

18. Scullen T, Keen J, Mathkour M, et al. Coronavirus 2019 (COVID-19)associated encephalopathies and cerebrovascular disease: the New Orleans experience. World Neurosurg 2020;141:e437-46 CrossRef Medline
19. Poudel GR, Dominguez JF, Verhelst H, et al. Network diffusion modeling predicts neurodegeneration in traumatic brain injury. Ann Clin Transl Neurol 2020;7:270-79 CrossRef Medline

20. Poudel GR, Harding IH, Egan GF, et al. Network spread determines severity of degeneration and disconnection in Huntington's disease. Hum Brain Mapp 2019;40:4192-4201 CrossRef Medline

21. Payus AO, Lin CL, Noh MM, et al. SARS-CoV-2 infection of the nervous system: a review of the literature on neurological involvement in novel coronavirus disease (COVID-19). Bosn J Basic Med Sci 2020;20:283-92 CrossRef Medline

22. DosSantos MF, Devalle S, Aran V, et al. Neuromechanisms of SARSCoV-2: a review. Front Neuroanat 2020;14:37 CrossRef Medline

23. Edlow BL, Takahashi E, Wu O, et al. Neuroanatomic connectivity of the human ascending arousal system critical to consciousness and its disorders. J Neuropathol Exp Neurol 2012;71:531-46 CrossRef Medline

24. Afshar H, Yassin Z, Kalantari S, et al. Evolution and resolution of brain involvement associated with SARS- CoV2 infection: a close clinical-paraclinical follow up study of a case. Mult Scler Relat Disord 2020;43:102216 CrossRef Medline

25. Ding J, Sigurðsson S, Jónsson PV, et al. Space and location of cerebral microbleeds, cognitive decline, and dementia in the community. Neurology 2017;88:2089-97 CrossRef Medline

26. Wang Y, Jiang Y, Suo C, et al. Deep/mixed cerebral microbleeds are associated with cognitive dysfunction through thalamocortical connectivity disruption: the Taizhou Imaging Study. Neuroimage Clin 2019;22:101749 CrossRef Medline

27. Bonardel C, Bonnerot M, Ludwig M, et al. Bilateral posterior cerebral artery territory infarction in a SARS-Cov-2 infected patient: discussion about an unusual case. J Stroke Cerebrovasc Dis 2020;29:105095 CrossRef Medline

28. Guillan M, Villacieros-Alvarez J, Bellido S, et al. Unusual simultaneous cerebral infarcts in multiple arterial territories in a COVID-19 patient. Thromb Res 2020;193:107-09 CrossRef Medline

29. Novi G, Rossi T, Pedemonte E, et al. Acute disseminated encephalomyelitis after SARS-CoV-2 infection. Neurol Neuroimmunol Neuroinflamm 2020;77:e797 CrossRef Medline

30. Netland J, Meyerholz DK, Moore S, et al. Severe acute respiratory syndrome coronavirus infection causes neuronal death in the absence of encephalitis in mice transgenic for human ACE2. J Virol 2008;82:7264-75 CrossRef Medline

31. Desforges M, Le Coupanec A, Dubeau P, et al. Human coronaviruses and other respiratory viruses: underestimated opportunistic pathogens of the central nervous system? Viruses 2019;12:14 CrossRef Medline

32. Bailey DM, Bärtsch $P$, Knauth $M$, et al. Emerging concepts in acute mountain sickness and high-altitude cerebral edema: from the molecular to the morphological. Cell Mol Life Sci 2009;66:3583-94 CrossRef Medline

33. Wang $Y$, Liu G, Hong $D$, et al. White matter injury in ischemic stroke. Prog Neurobiol 2016;141:45-60 CrossRef Medline

34. Martinez-Ramirez S, Greenberg SM, Viswanathan A. Cerebral microbleeds: overview and implications in cognitive impairment. Alzheimers Res Ther 2014;6:33 CrossRef Medline

35. Agarwal S, Jain R, Dogra S, et al. Cerebral microbleeds and leukoencephalopathy in critically ill patients with COVID-19. Stroke 2020;51:2649-55 CrossRef Medline

36. Lu Y, Li X, Geng D, et al. Cerebral micro-structural changes in COVID-19 patients: an MRI-based 3-month follow-up study. EClinicalMedicine 2020;25:100484 CrossRef Medline 